\title{
Applications of dual MRM for determining the natural frequencies and natural modes of a rod using the singular value decomposition method
}

\author{
W. Yeih ${ }^{\mathrm{a}}$, J.R. Chang ${ }^{\mathrm{b}}$, C.M. Chang ${ }^{\mathrm{a}}$, J.T. Chen ${ }^{\mathrm{a}, *}$ \\ ${ }^{a}$ Department of Harbor and River Engineering, National Taiwan Ocean University, Keelung 202, Taiwan \\ ${ }^{\mathrm{b}}$ Department of Naval Architecture, National Taiwan Ocean University, Keelung 202, Taiwan
}

Received 14 September 1998; accepted 1 December 1998

\begin{abstract}
In this article, the dual multiple reciprocity method is employed to solve the natural frequencies and natural modes for a rod. The conventional approach using dual MRM is not qualified as a systematic method because of the following two reasons: (1) it needs to distinguish the spurious eigenvalue only after the corresponding eigenmode is obtained; (2) the possible indeterminancy of eigenvector may be encountered when the constraint equations chosen are highly dependent such that the rank of the leading coefficient matrix is insufficient. To construct a systematic way, we propose to consider all constraint equations together instead of using the singular or hypersingular equation alone as the conventional MRM uses. The singular value decomposition method is, then, used to solve the eigenproblem after combining the singular and hypersingular equations. This method can avoid the spurious eigenvalue problem and the possible indeterminancy of boundary eigenvectors at the same time. Three numerical examples are given to verify the validity of the present method. (C) 1999 Elsevier Science Ltd. All rights reserved.
\end{abstract}

Keywords: Dual multiple reciprocity method; Rod; Natural modes; Singular value decomposition

\section{Introduction}

The vibration of structures is extremely important in applied engineering. The free vibration problem is the most fundamental problem in studying vibration as the eigenfrequency of the homogeneous differential equation system is the characteristic frequency (natural frequency) of the structure and one should avoid having an excitation source with this characteristic frequency acting on the structure to result in resonance. Furthermore, the natural modes obtained from the free vibration problem actually represent the solution basis of the forced vibration problem, (i.e., the solution of the forced vibration problem) (nonhomogeneous differential equation) can be constructed by the summation of the products of the natural modes (basis functions) and projections (generalized Fourier coefficients). For the reason stated earlier, the free vibration problem is usually taken as the first step to tackle the wonder world of vibration. The free vibration problem is often modelled as a Helmholtz equation in the frequency domain. To solve the Helmholtz equation numerically, either the finite element method or the boundary element method can be available.

\footnotetext{
* Corresponding author. Fax: +886-2-462-2192.

E-mail address: b0209@ntou66.ntou.edu.tw (J.T. Chen)
}

To solve a Helmholtz equation using the boundary element approach, the complex-valued fundamental solution has been employed to solve eigenproblems [1,2]. This is a natural choice as the operator is the Helmholtz equation. However, two difficulties may be encountered using such approach: (1) computation in the domain of a complex number needs much efforts comparing with that in the domain of a real number; (2) the wave number is embedded in the fundamental solution so that one has to calculate the influence matrix every time at different wave number. As a result of these two reasons, the boundary element method is sometimes not recommended to solve such an eigenproblem.

To avoid computation in the domain of a complex number, the multiple reciprocity method (MRM) has been employed to solve the Helmholtz problem in the real domain [3-5]. In this algorithm, the Helmholtz equation is treated as a Poisson equation with an external source; therefore, the fundamental solution of the Laplace equation is considered. However, the domain integral is present as a result of the integration of the external source. MRM can transform this domain integral into boundary integrals iteratively such that the domain cell is not needed when the remainder terms of the domain integral can be neglected. Moreover, the MRM separates the wave number from the 
influence matrix calculation such that one needs not to recompute the influence matrix at different wave number. In the literature, the conventional singular integral equation (UT equation) was used only in MRM [3]. Chen et al. [6] found that the conventional MRM using the singular integral equation results in spurious eigenvalues by providing 1$D$ rod example. In addition, boundary eigenvectors may not be found when the rank of the leading coefficient matrix is insufficient. To filter out the spurious eigenvalues, they proposed to construct the second set of equations, the hypersingular integral equation (LM equation). By using the idea that the corresponding spurious eigenmode obtained by UT equation cannot satisfy LM equation and vice versa, they successfully filtered out the spurious eigenvalues. As to avoid the possible indeterminancy of boundary eigenvector, when the rank of the leading coefficient matrix obtained from UT or LM equation is insufficient, they proposed to replace some constraint equations in the original equation set (UT or LM) by those in the complementary equation set (LM or UT equation). Their leading efforts did not only explore the drawbacks of MRM but also propose the dual MRM to deal with them. In brief words, only dual MRM (UT + LM equations) can provide enough constraint equations. The dual BEM structure, which uses the singular integral and hypersingular integral equations together, has been well recognized as the key of providing enough constraint equations in boundary value problem with a degenerate boundary [7-9].

Kamiya et al. [10] found that the kernels in MRM are no more than real parts of the kernels in the complex-valued formulation for the two-dimensional case. Yeih et al. [11] proved that MRM can be constructed such that it is fully equivalent to the complex-valued formulation by adding a complex constant into the zeroth fundamental solution for the Laplace operator when the radiation condition is satisfied. Furthermore, they clearly explained why the spurious eigenvalue problem is encountered in the conventional dual MRM.

In this article, the problem of determining natural frequencies and natural modes using the MRM is revisited. We propose a new methodology to modify Chen's work [6]. A brief review of the dual MRM is first introduced for readers' convenience. Then, the difficulties encountered in Chen's method are also stated. To solve the eigenproblem more efficiently, the singular value decomposition method (SVD) is adopted. The SVD method can avoid the spurious eigenvalue problem at the stage of eigenvalue searching and find the boundary eigenvector more efficiently in the sense of the least square error; therefore, it qualifies as a systematic solution method. Three examples have been solved using the dual MRM, and the results demonstrate the validity of the current research. As the proposed method is independent of dimension, we begin our study from one dimensional problems because their analytical solutions are available. This work is a prelude of higher dimensional problems.

\section{Problem statement and analytical derivations}

For simplicity, we consider a one-dimensional rod vibration problem with the following governing equation:

$$
\frac{\mathrm{d}^{2} u(x)}{\mathrm{d} x^{2}}+\lambda u(x)=0, \quad 0 \leq x \leq 1,
$$

where $\lambda$ and $u(x)$ denote the eigenvalue and eigenmode, respectively. Without loss of generality, it is assumed that the rod has a unit length.

Three benchmark examples are considered as follows [6]:

Case 1: $u(0)=0, u(1)=0$ (Dirichlet B.C.),

Case 2: $t(0)=0, t(1)=0$ (Neumann B.C.),

Case 3: $u(0)=0, t(1)=0$ (Mixed B.C. $)$,

where

$t\left(x_{0}\right)=\left.\frac{\mathrm{d} u(x)}{\mathrm{d} x}\right|_{x=x_{0}}$.

Consider an auxilliary system with a fundamental solution satisfying

$\frac{\mathrm{d}^{2} U(x, s)}{\mathrm{d} x^{2}}=\delta(x-s), \quad-\infty<x<\infty$,

where $U(x, s)$ is a fundamental solution expressed as

$U(x, s)=\frac{1}{2}|x-s|=U^{(0)}(x, s)$.

In the earlier expression, $U^{(0)}(x, s)$ is called the zerothorder fundamental solution.

By employing Green's third identity, we have

$$
\begin{aligned}
& \int_{0}^{1} \nabla^{2} U^{(0)}(x, s) u(x) \mathrm{d} x=\int_{0}^{1} U^{(0)}(x, s) \nabla^{2} u(x) \mathrm{d} x \\
& \left.+\left[u(x) \frac{\mathrm{d} U^{(0)}(x, s)}{\mathrm{d} x}-\frac{\mathrm{d} u(x)}{\mathrm{d} x} U^{(0)}(x, s)\right]\right]_{x=0}^{x=1} .
\end{aligned}
$$

By transforming the domain integral term into boundary integral terms, the primary and secondary fields for $u(s)$ and $t(s)$ can be expressed as

$$
\begin{aligned}
u(s) & =\left\{u(x) T^{(0)}(x, s)-t(x) U^{(0)}(x, s)\right. \\
+ & \left.\sum_{j=0}^{N}\left[b^{(j)} T^{(j+1)}(x, s)-\frac{\mathrm{d} b^{(j)}}{\mathrm{d} x} U^{(j+1)}(x, s)\right]\right\}\left.\right|_{x=0} ^{x=1} \\
& +R_{N+1}, \\
t(s) & =\left\{u(x) M^{(0)}(x, s)-t(x) L^{(0)}(x, s)\right. \\
& \left.\left.+\sum_{j=0}^{N}\left[b^{(j)} M^{(j+1)}(x, s)-\frac{\mathrm{d} b^{(j)}}{\mathrm{d} x} L^{(j+1)}(x, s)\right]\right\}\right\}_{x=0}^{x=1}+R_{N+1}^{\prime},
\end{aligned}
$$


Table 1

Explicit forms for the kernel functions $(\boldsymbol{r}=|\boldsymbol{x}-\boldsymbol{s}|)$

\begin{tabular}{lll}
\hline Kernel & $\mathrm{x}>\mathrm{s}$ & $\mathrm{x}<\mathrm{s}$ \\
$U^{(j+1)}(x, s)$ & $\frac{1}{2} \frac{r^{(2 j+3)}}{(2 j+3) !}(j \geq-1)$ & $-\frac{1}{2} \frac{r^{(2 j+3)}}{(2 j+3) !}(j \geq-1)$ \\
$T^{(j+1)}(x, s)$ & $\frac{1}{2} \frac{r^{(2 j+2)}}{(2 j+2) !}(j \geq-1)$ & $-\frac{1}{2} \frac{r^{(2 j+2)}}{(2 j+2) !}(j \geq-1)$ \\
$L^{(j+1)}(x, s)$ & $-\frac{1}{2} \frac{r^{(2 j+2)}}{(2 j+2) !}(j \geq-1)$ & $\frac{1}{2} \frac{r^{(2 j+2)}}{(2 j+2) !}(j \geq-1)$ \\
$M^{(j+1)}(x, s)$ & $-\frac{1}{2} \frac{r^{(2 j+1)}}{(2 j+1) !}(j \geq 0)$ & $\frac{1}{2} \frac{r^{(2 j+1)}}{(2 j+1) !}(j \geq 0)$ \\
& $0(j=-1)$ & $0(j=-1)$
\end{tabular}

where $R_{N+1}^{\prime}$ is the derivative of $R_{N+1}$ with respect to $s$, and the explicit forms for the kernel functions are shown in Table 1 and defined as

$T^{(j+1)}(x, s)=\frac{\partial\left\{U^{(j+1)}(x, s)\right\}}{\partial x}$,

$L^{(j+1)}(x, s)=\frac{\partial\left\{U^{(j+1)}(x, s)\right\}}{\partial s}$,

$M^{(j+1)}(x, s)=\frac{\partial^{2}\left\{U^{(j+1)}(x, s)\right\}}{\partial x \partial s}$

Eqs. (5) and (6) comprise the dual equations for MRM.

By moving the field point close to the boundary, the dual BEM can be derived as follows:

$T_{0} \tilde{u}-U_{0} \tilde{t}=\sum_{i=1}^{N}\left(T_{i}(\lambda) \tilde{u}-U_{i}(\lambda) \tilde{t}\right)$

$M_{0} \tilde{u}-L_{0} \tilde{t}=\sum_{i=1}^{N}\left(M_{i}(\lambda) \tilde{u}-L_{i}(\lambda) \tilde{t}\right)$,

where $\tilde{u}$ and $\tilde{t}$ are the column vectors of the boundary data. Substituting the values of the kernel functions shown in Table 1 into Eqs. (10) and (11), we have

$\left[\begin{array}{cc}1 / 2 & -1 / 2 \\ -1 / 2 & 1 / 2\end{array}\right]\left\{\begin{array}{l}u(0) \\ u(1)\end{array}\right\}-\left[\begin{array}{cc}0 & -1 / 2 \\ 1 / 2 & 0\end{array}\right]\left\{\begin{array}{l}t(0) \\ t(1)\end{array}\right\}$

$=\left[\begin{array}{cc}0 & \sum_{j=0}^{\infty} \frac{1}{2} \frac{(-\lambda)^{j+1}}{(2 j+2) !} \\ \sum_{j=0}^{\infty} \frac{1}{2} \frac{(-\lambda)^{j+1}}{(2 j+2) !} & 0\end{array}\right]\left\{\begin{array}{l}u(0) \\ u(1)\end{array}\right\}$

$-\left[\begin{array}{cc}0 & \sum_{j=0}^{\infty} \frac{1}{2} \frac{(-\lambda)^{j+1}}{(2 j+3) !} \\ -\sum_{j=0}^{\infty} \frac{1}{2} \frac{(-\lambda)^{j+1}}{(2 j+3) !} & 0\end{array}\right]\left\{\begin{array}{l}t(0) \\ t(1)\end{array}\right\}$ and

$$
\begin{aligned}
& {\left[\begin{array}{ll}
0 & 0 \\
0 & 0
\end{array}\right]\left\{\begin{array}{l}
u(0) \\
u(1)
\end{array}\right\}-\left[\begin{array}{cc}
\frac{-1}{2} & \frac{1}{2} \\
\frac{1}{2} & \frac{-1}{2}
\end{array}\right]\left\{\begin{array}{l}
t(0) \\
t(1)
\end{array}\right\}} \\
& =\left[\begin{array}{cc}
0 & -\sum_{j=0}^{\infty} \frac{1}{2} \frac{(-\lambda)^{j+1}}{(2 j+1) !}
\end{array}\right\}\left\{\begin{array}{l}
u(0) \\
u(1)
\end{array}\right\} \\
& -\left[\begin{array}{c}
\sum_{j=0}^{\infty} \frac{1}{2} \frac{(-\lambda)^{j+1}}{(2 j+1) !} \\
-\sum_{j=0}^{\infty} \frac{1}{2} \frac{(-\lambda)^{j+1}}{(2 j+2) !}
\end{array}\right]\left\{\begin{array}{l}
t(0) \\
t(1)
\end{array}\right\} .
\end{aligned}
$$

After summing the infinite series in Eqs. (12) and (13), the three terms can be summed into a closed-form function:

$1+\sum_{j=0}^{\infty} \frac{(-\lambda)^{j+1}}{(2 j+3) !}=\frac{\sin \sqrt{\lambda}}{\sqrt{\lambda}}$

$1+\sum_{j=0}^{\infty} \frac{(-\lambda)^{j+1}}{(2 j+2) !}=\cos \sqrt{\lambda}$

$\sum_{j=0}^{\infty} \frac{(-\lambda)^{j+1}}{(2 j+1) !}=-\sqrt{\lambda} \sin \sqrt{\lambda}$

Eqs. (10) and (11) then can be rewritten as
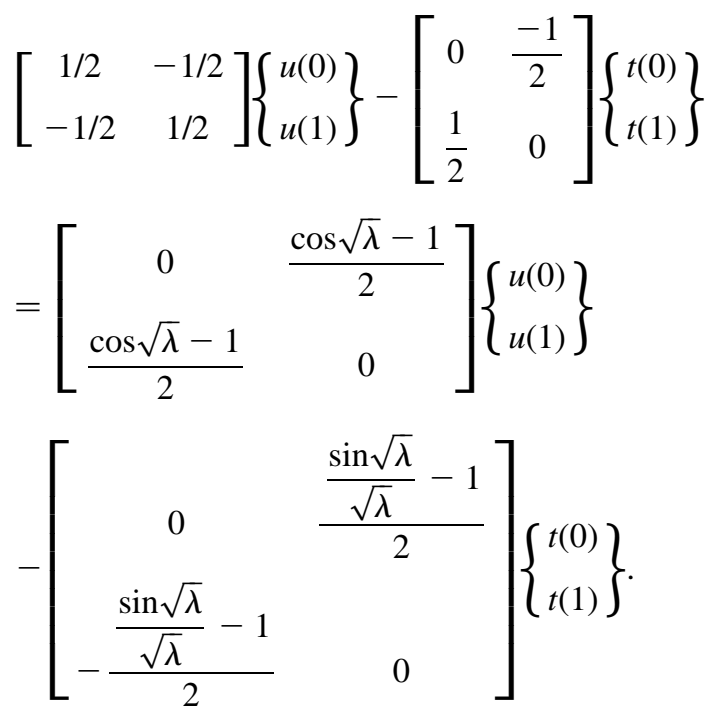
Table 2

Eigensolutions for the Dirichlet problem (case 1)

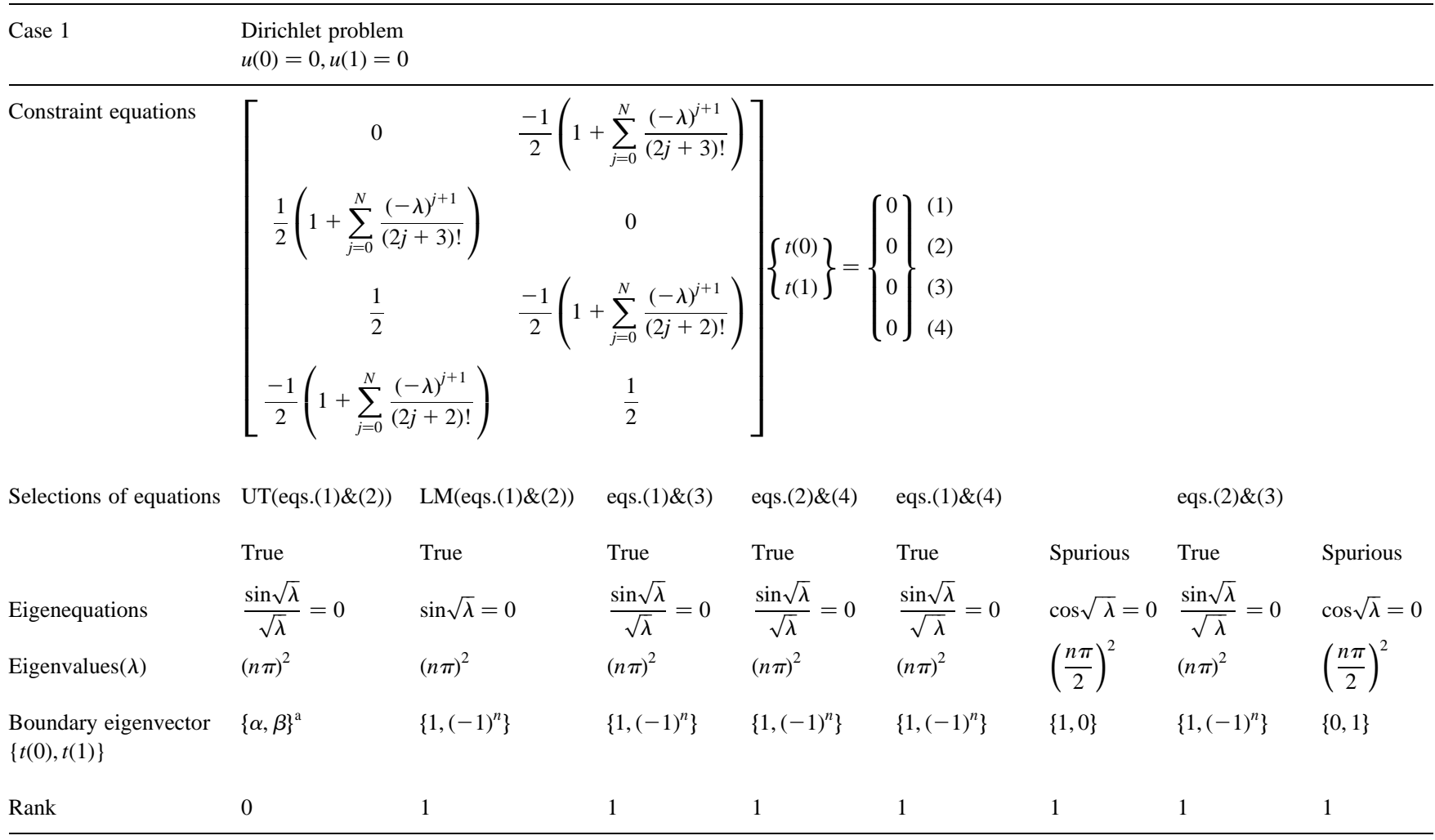

${ }^{\mathrm{a}}$ where $\alpha$ and $\beta$ are arbitrary constants.

Table 3

Eigensolutions for the Neumann problem (case 2)

Case $2 \quad$ Neumann problem $t(0)=0, t(1)=0$

Constraint equations

$\left.\left[\begin{array}{cc}\frac{1}{2} & \frac{-1}{2}\left(1+\sum_{j=0}^{N} \frac{(-\lambda)^{j+1}}{(2 j+2) !}\right) \\ \frac{-1}{2}\left(1+\sum_{j=0}^{N} \frac{(-\lambda)^{j+1}}{(2 j+2) !}\right) & \frac{1}{2} \\ 0 & \frac{1}{2} \sum_{j=0}^{N} \frac{(-\lambda)^{j+1}}{(2 j+1) !} \\ \frac{-1}{2} \sum_{j=0}^{N} \frac{(-\lambda)^{j+1}}{(2 j+1) !}\end{array}\right]\left\{\begin{array}{l}u(0) \\ u(1)\end{array}\right\}=\left\{\begin{array}{l}0 \\ 0 \\ 0 \\ 0\end{array}\right\} \begin{array}{l}(1) \\ (3) \\ (4)\end{array}\right]$

\begin{tabular}{|c|c|c|c|c|c|c|c|c|}
\hline \multirow[t]{2}{*}{ Selections of equations } & UT.(eqs.(1)\&(2)) & LM(eqs.(1)\&(2)) & eqs.(1)\&(3) & eqs.(2)\&(4) & eqs.(1)\&(4) & \multicolumn{3}{|c|}{ eqs.(2)\&(3) } \\
\hline & True & True & True & True & True & Spurious & True & Spurious \\
\hline Eigenequations & $\sin \sqrt{\lambda}=0$ & $\sqrt{\lambda} \sin \sqrt{\lambda}=0$ & $\sqrt{\lambda} \sin \sqrt{\lambda}=0$ & $\sqrt{\lambda} \sin \sqrt{\lambda}=0$ & $\sqrt{\lambda} \sin \sqrt{\lambda}=0$ & $\cos \sqrt{\lambda}=0$ & $\sqrt{\lambda} \sin \sqrt{\lambda}=0$ & $\cos \sqrt{\lambda}=0$ \\
\hline Eigenvalues $(\lambda)$ & $(n \pi)^{2}$ & $(n \pi)^{2}$ & $(n \pi)^{2}$ & $(n \pi)^{2}$ & $(n \pi)^{2}$ & $\left(\frac{n \pi}{2}\right)^{2}$ & $(n \pi)^{2}$ & $\left(\frac{n \pi}{2}\right)^{2}$ \\
\hline $\begin{array}{l}\text { Boundary eigenvector } \\
\{u(0), u(1)\}\end{array}$ & $\left\{1,(-1)^{n}\right\}$ & $\{\alpha, \beta\}^{\mathrm{a}}$ & $\left\{1,(-1)^{n}\right\}$ & $\left\{1,(-1)^{n}\right\}$ & $\left\{1,(-1)^{n}\right\}$ & $\{0,1\}$ & $\left\{1,(-1)^{n}\right\}$ & $\{1,0\}$ \\
\hline rank & 1 & 0 & 1 & 1 & 1 & 1 & 1 & 1 \\
\hline
\end{tabular}

${ }^{\text {a }}$ where $\alpha$ and $\beta$ are arbitrary constants. 


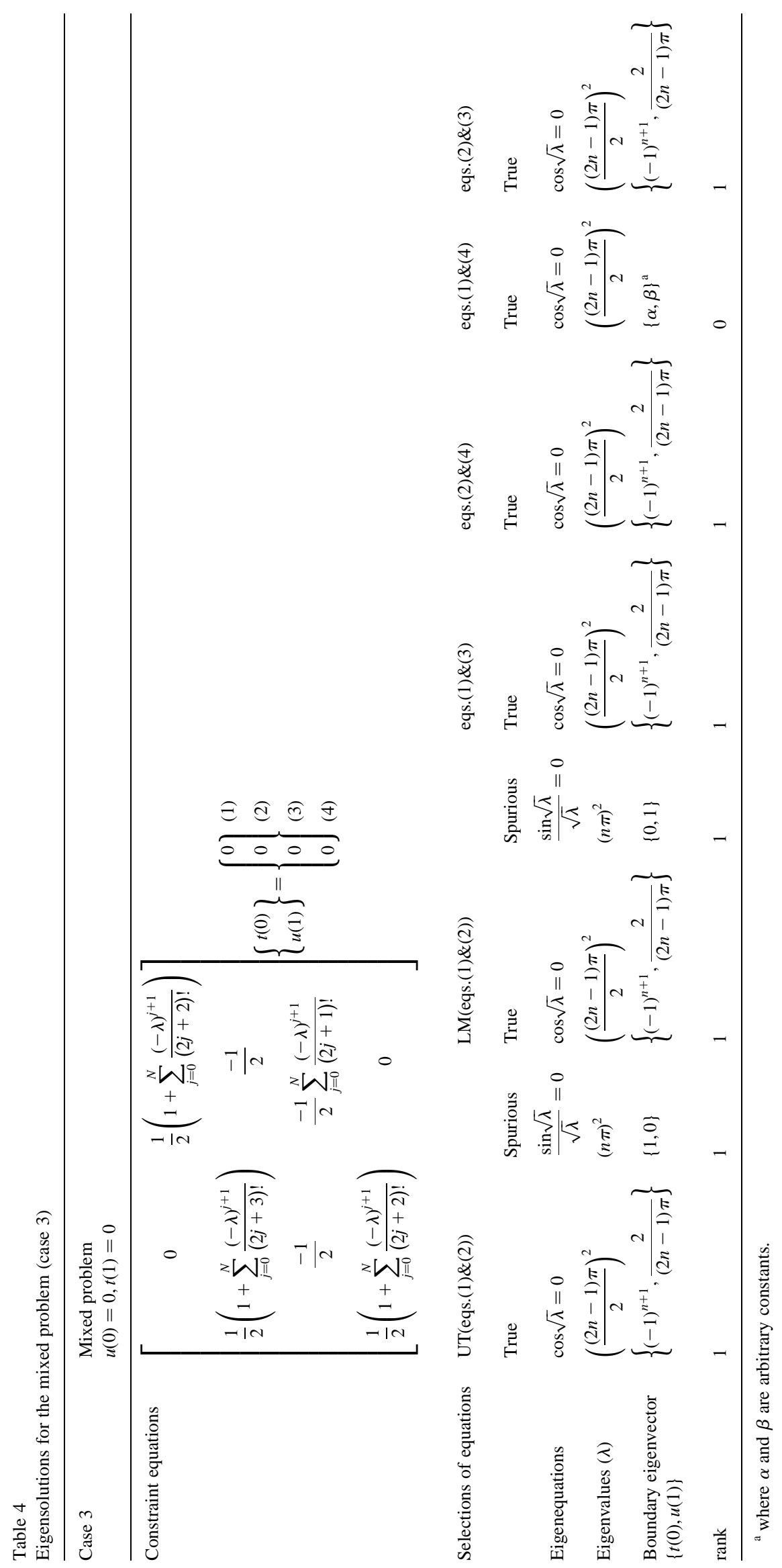


and

$\left[\begin{array}{ll}0 & 0 \\ 0 & 0\end{array}\right]\left\{\begin{array}{l}u(0) \\ u(1)\end{array}\right\}-\left[\begin{array}{cc}-1 / 2 & 1 / 2 \\ 1 / 2 & -1 / 2\end{array}\right]\left\{\begin{array}{l}t(0) \\ t(1)\end{array}\right\}$

$=\left[\begin{array}{cc}0 & -\frac{\sqrt{\lambda} \sin \sqrt{\lambda}}{2} \\ \frac{\sqrt{\lambda} \sin \sqrt{\lambda}}{2} & 0\end{array}\right]\left\{\begin{array}{l}u(0) \\ u(1)\end{array}\right\}$

$-\left[\begin{array}{cc}0 & -\frac{\cos \sqrt{\lambda}-1}{2} \\ -\frac{\cos \sqrt{\lambda}-1}{2} & 0\end{array}\right]\left\{\begin{array}{c}t(0) \\ t(1)\end{array}\right\}$.

\section{Difficulties encountered using the singular or hypersingular integral equations alone}

After substituting the boundary conditions of cases (1), (2) and (3) into Eqs. (12) and (13), the eigenequations, eigenvalues and boundary eigenvector can be obtained as shown in Tables 2-4.

After obtaining the boundary eigenvector, the representation for the displacement field can be expressed as follows:

$u(s)=\left[\begin{array}{lll}H_{1}(s) & H_{2}(s) \quad H_{3}(s) \quad H_{4}(s)\end{array}\right]\left\{\begin{array}{c}u(0) \\ u(1) \\ t(0) \\ t(1)\end{array}\right\}$,

$0 \leq s \leq 1$

where

$H_{1}(s) \equiv \frac{1}{2} \cos (\sqrt{\lambda} s)$,

$H_{2}(s) \equiv \frac{1}{2} \cos (\sqrt{\lambda}(1-s))$

$H_{3}(s) \equiv \frac{\sin (\sqrt{\lambda} s)}{2 \sqrt{\lambda}}$,

$H_{4}(s) \equiv-\frac{\sin (\sqrt{\lambda}(1-s))}{2 \sqrt{\lambda}}$.

Even when eigenvalues can be determined for case (1) and case (2) using the UT or LM equation, the selections of independent equations with rank of 1 to determine the boundary mode are not unique as shown in Tables 2 and 3. It is found that the UT equation cannot be used alone to determine the eigenmodes for case (1) with the Dirichelet data shown in Table 2; however, the LM equation also cannot be used alone to determine the eigenmodes for case (2) with the Neumann data shown in Table 3. Chen et al. [6] proposed use of the complementary equation in the dual BEM framework to determine the boundary mode when the UT (in case (1)) or LM (in case (2)) equation fails to determine the boundary mode. UT and LM equations make the rank of the leading coefficient matrix equal to 1 ; thus, the boundary eigenvector can be determined. However, this method is inconvenient as many different combinations of equations must be tried manually in order to find the combination which can obtain the boundary mode.

It can be found from Table 4 that spurious eigenvalues exist in the case of the mixed boundary condition. Chen et al. [6] proposed combining UT and LM equations together to filter out the spurious eigenvalues. As UT and LM equations are both constraint equations that the boundary data should satisfy, it is easy to filter out spurious eigenvalues by examining if the boundary eigenmodes obtained using the UT equation can satisfy the constraint equations of the LM equation and vice versa. It is found that the only "eigenmode" satisfying both the UT and LM equation at the spurious eigenvalue is the trivial eigenmode, so it is concluded that this eigenvalue should be spurious.

Chen's method, although successful in filtering out spurious eigenvalues, requires that the boundary eigenmode at the spurious eigenvalues be solved first, which means that their method cannot filter out the spurious eigenvalues in the stage of eigenvalue searching. Let us summarize the difficulties encountered in Chen's method [6]: (1) the boundary eigenmode at the true eigenvalues may possibly be undetermined; (2) the spurious eigenvalue needs to be filtered out only at the stage when the corresponding spurious eigenmode is obtained.

It is quite interesting to ask ourselves a question: can one filter out the spurious eigenvalues in the stage of eigenvalue searching and determine the boundary eugenmodes at the true eigenvalues more systemmatically and efficiently? The answer is yes, as will be shown in the following section.

\section{Determination of the eigenproblem using the SVD method}

After substituting the homogeneous boundary conditions for both the UT and LM integral equations into three examples (Case 1: $u(0)=0, u(1)=0$, Case 2: $t(0)=0, t(1)=0$, Case 3: $u(0)=0, t(1)=0)$, the eigenproblem in general can be expressed as

$[\boldsymbol{A}(\lambda)]_{2 \times 2} \boldsymbol{x}_{2 \times 1}=0$,

where $\boldsymbol{A}(\lambda)$ is the leading coefficient matrix function of $\lambda$, and $\boldsymbol{x}$ is the boundary eigenvector. When $\lambda$ is equal to the eigenvalue, the determinant of $\mathbf{A}$ is zero, which means that the rank of $\mathbf{A}$ must be at most equal to one in order to have a nontrivial solution. We should remember that we have in total four equations (UT + LM equations). Therefore, the 


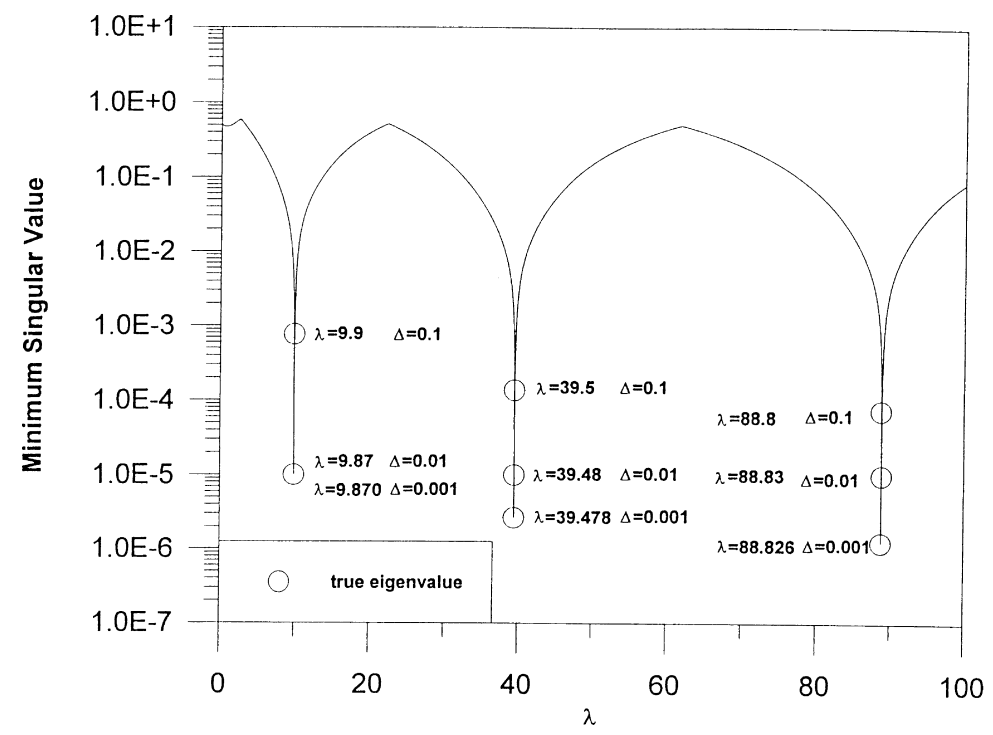

Fig. 1. Direct search of eigenvalues using SVD for the rod case (Dirichlet B.C.: $u(0)=u(1)=0, n=20$ )

boundary eigenvectors should satisfy all four equations even though we obtain the eigenvalues from the two selected equations independently. It has been mentioned earlier that the spurious boundary eigenvectors corresponding to the spurious eigenvalues cannot satisfy the remaining eigenequations. This means that the only eigensolution corresponding to the spurious eigenvalues is a null vector as the leading coefficient matrix after combining the UT and LM equations has a rank of 2 . In another words, a selection of two equations which has a rank of 2 is possible. However, when we select a special group of equations, the rank of the system of equations may be reduced by one order, which may mislead us and cause us to believe that it is a true eigenvalue.

To determine the boundary eigenvector $\boldsymbol{x}$, a standard procedure is to let one element in the vector of $\boldsymbol{x}$ be equal to one and reduce the equation to

$[\bar{A}]_{1 \times 1} \bar{x}_{1 \times 1}=b_{1 \times 1}$.

Then, the remaining components in the boundary eigenvector can be determined. However, the previously-mentioned algorithm is correct only when the rank of the leading coefficient matrix, $\boldsymbol{A}$, is equal to 1 . When the rank of the leading coefficient matrix is less than 1 , the algorithm fails.

In the rod case with the Dirichlet boundary conditions $u(0)=u(1)=0$, it is found that the rank of the leading coefficient matrix in the UT equation is equal to zero. In a similar way, for the case of $t(0)=t(1)=0$, it is found that the rank of the leading coefficient matrix in the LM equation is equal to zero. This means that the system of equations for case (1) appearing in the UT equation is highly dependent, so that the boundary eigenvector can be chosen arbitrarily. In general, the true boundary eigenvector should satisfy all four equations. Therefore, we have more equations than unknowns in this framework if UT and LM equations are both considered.
As mentioned earlier, the conventional method for finding the eigenvalues and corresponding boundary eigenvectors may encounter two difficulties: the spurious eigenvalue problem and the indeterminancy of boundary eigenvectors. Here, we propose use of the singular value decomposition method (SVD) to eliminate these two difficulties at the same time. A brief introduction to SVD is given now.

Consider a linear algebra problem with more equations than unknowns:

$[\boldsymbol{A}]_{m \times n} \boldsymbol{x}_{n \times 1}=\boldsymbol{b}_{m \times 1}, \quad m>n$,

where $m$ is the number of equations, $n$ is the number of unknowns and $\mathbf{A}$ is the leading matrix, which can be decomposed into

$[\boldsymbol{A}]_{m \times n}=\boldsymbol{U}_{m \times m} \boldsymbol{\Sigma}_{m \times n} \boldsymbol{V}_{n \times n}^{*}$.

Here, $\boldsymbol{U}$ is a left unitary matrix constructed by the left singular vectors, $\boldsymbol{\Sigma}$ is a diagonal matrix which has singular values $\sigma_{1}, \sigma_{2}, \cdots, \sigma_{n}$ allocated in a diagonal line as

$\Sigma=\left[\begin{array}{ccc}\sigma_{1} & \cdots & 0 \\ \vdots & \ddots & \vdots \\ 0 & \cdots & \sigma_{n} \\ \vdots & \ddots & \vdots \\ 0 & \cdots & 0\end{array}\right], \quad m>n$,

in which $\sigma_{1} \geq \sigma_{2} \cdots \geq \sigma_{n}$, and $V^{*}$ is the complex conjugate transpose of a right unitary matrix constructed by the right singular vectors. As we can see in Eq. (27), there exist at most $n$ nonzero singular values. This means that we can find at most $n$ linear independent equations in the system of equations. If we have $l$ zero singular values $(0 \leq l \leq n)$, this means that the rank of the system of equations is equal to $n-l$. However, the singular value may be very 


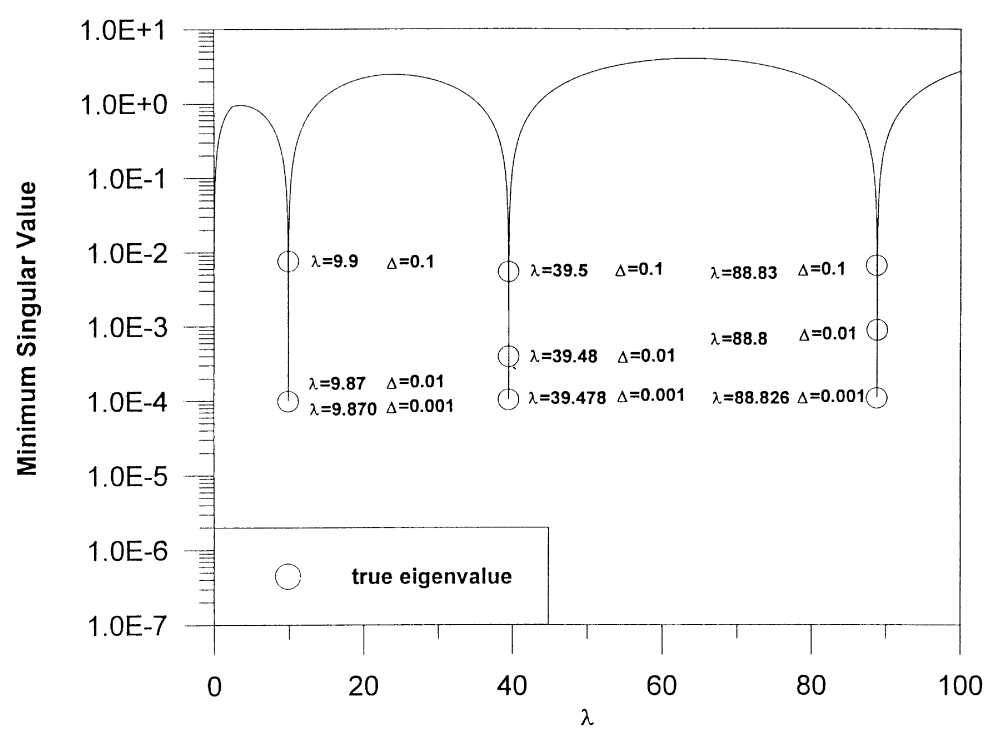

Fig. 2. Direct search of eigenvalues using SVD for the rod case (Neumann B.C.: $t(0)=t(1)=0, n=20$ ).

close to zero numerically, resulting in rank deficiency. For a general eigenproblem as shown in this article, the eigenvalues will cause the rank to be $n-1$ (that is, 1).

Determining the eigenvalues of the system of equations has now been transformed into finding the values of $\lambda$ which make the rank of the leading coefficient matrix to be 1 . This means that when $m=4, n=2$ and $b_{2 \times 1}=0$, the eigenvalues will make $s=1$, such that the minimum singular value must be zero or very close to zero.

Let us take the rod as an example; the results obtained using the direct search method based on the SVD method are plotted in Figs. 1-3. It is seen that the true eigenvalues cause the minimum singular value to be much lower than the other $\lambda$ values. The more the eigenvalue approaches the true value, the more the minimum singular value approaches zero, in which case the rank of the matrix will be reduced to
1. However, this is not true for the spurious case. Therefore, the rank of the matrix is equal to 2 at the spurious eigenvalue, so the spurious eigenvalue can be easily distinguished and filtered out without need of any information from the boundary modes.

To find the boundary eigenvector associated with the eigenvalue, we can set one of the elements in the boundary eigenvector to be one and then reduce the equations into the form of Eq. (25), where $\boldsymbol{b}$ is now a nontrivial vector, $m=4$ and $n=1$. Then, the pseudo-inverse matrix, $\boldsymbol{A}^{+}$of $\boldsymbol{A}$, is expressed as

$$
\boldsymbol{A}_{n \times m}^{+}=\boldsymbol{V}_{n \times n} \boldsymbol{\Sigma}_{n \times m}^{+} \boldsymbol{U}_{m \times m}^{*},
$$

where $\boldsymbol{\Sigma}^{+}$is constructed by taking the transpose of $\boldsymbol{\Sigma}$ and then replacing the diagonal singular value terms with its

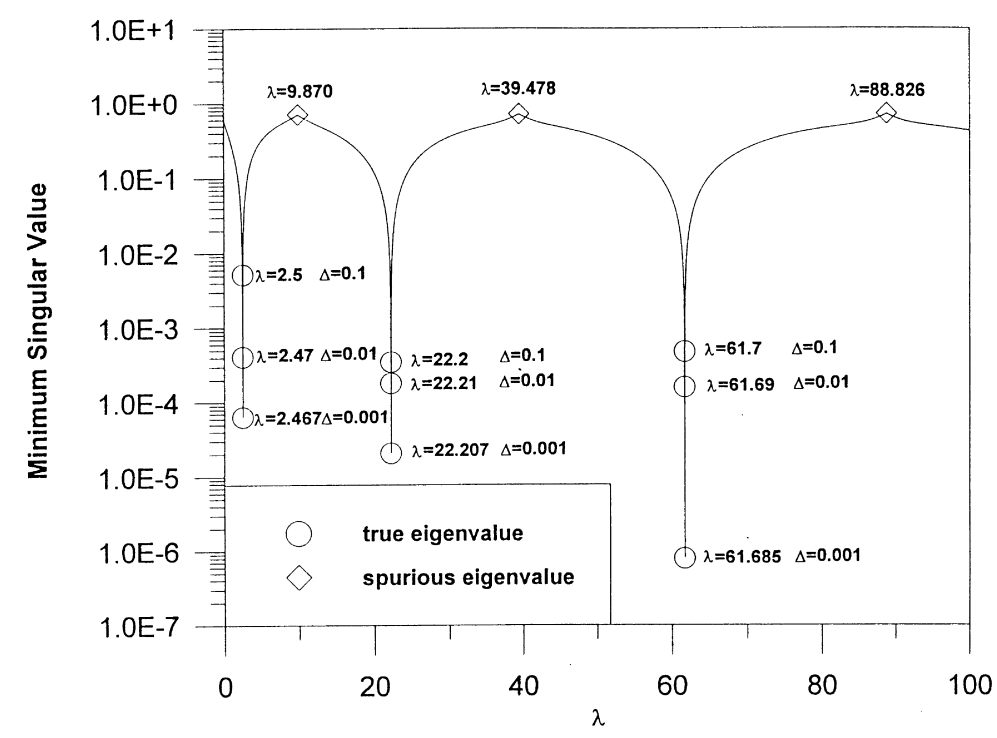

Fig. 3. Direct search of eigenvalues using SVD for the rod case (Mixed B.C.: $u(0)=t(1)=0, n=20$ ). 


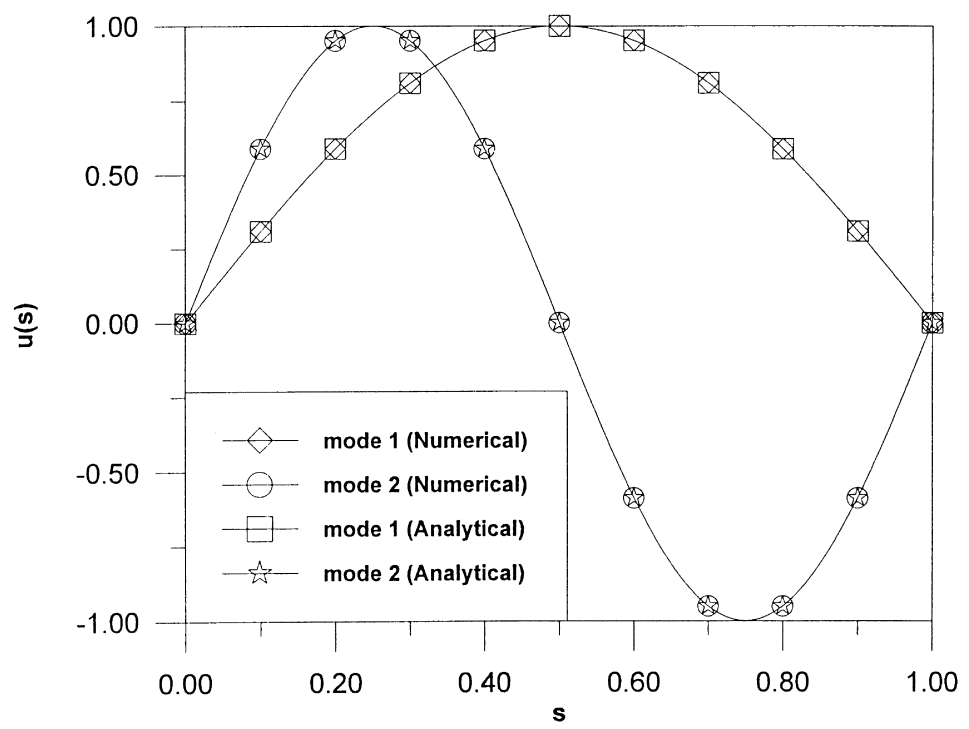

Fig. 4. The first two eigenmodes determined using the SVD method (Dirichlet B.C.: $u(0)=u(1)=0, n=20$ ).

inverse, expressed as

$\boldsymbol{\Sigma}^{+}=\left[\begin{array}{ccccc}\frac{1}{\sigma_{1}} & \cdots & 0 & \cdots & 0 \\ \vdots & \ddots & \vdots & \ddots & \vdots \\ 0 & \cdots & \frac{1}{\sigma_{n}} & \cdots & 0\end{array}\right] m>n$.

The previously-mentioned SVD method has been proved to be equivalent to the least square error solution in determining the unknown vector when the number of equations is larger than that of unknowns [12]. After introducing the SVD method, we do not need to worry about how to pick a specific group of equations such that the rank of the leading coefficient is sufficient to solve for the eigenvector. However, we can take all four equations into account, which apparently causes the rank of the leading coefficient matrix to be equal to one. Thus, the eigenvector can be easily found in the sense of the least square error. The eigenmodes determined using the SVD method are the same as those obtained in Chen's work [6] as the boundary eigenvectors are almost the same; therefore, only the first two mode shapes for the rod obtained using the SVD method are illustrated in Figs. 4-6. It can be confirmed that the SVD method can deal with the spurious eigenvalue problem and eliminate possible indeterminancy of the boundary eigenvector at the same time. For further details concerning the SVD method, please refer to Gloub's book [13].

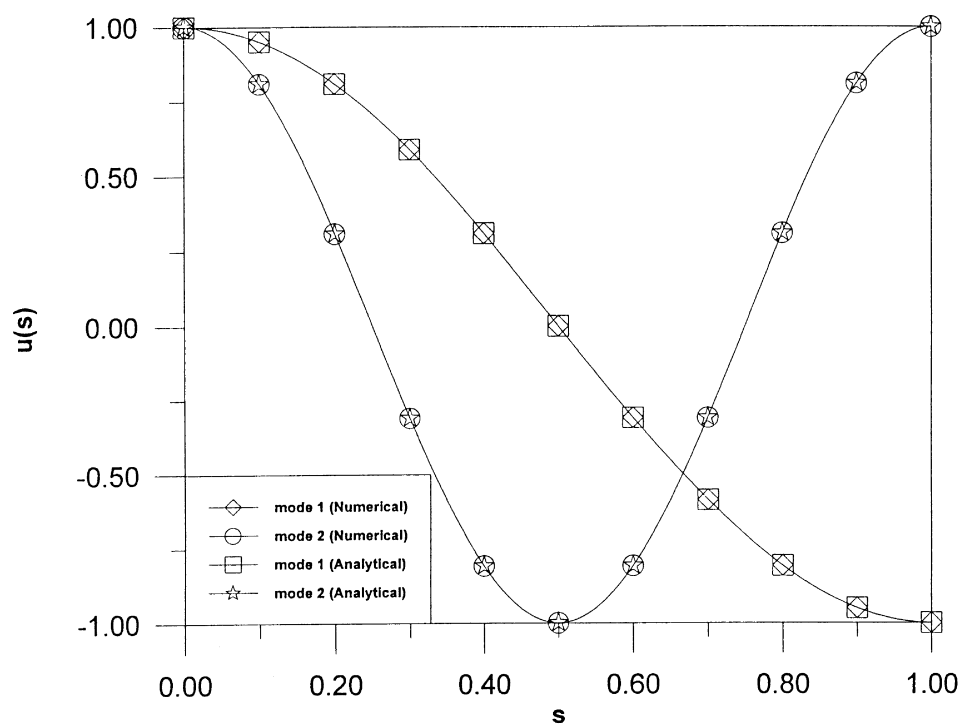

Fig. 5. The first two eigenmodes determined using the SVD method (Neumann B.C.: $t(0)=t(1)=0, n=20$ ). 


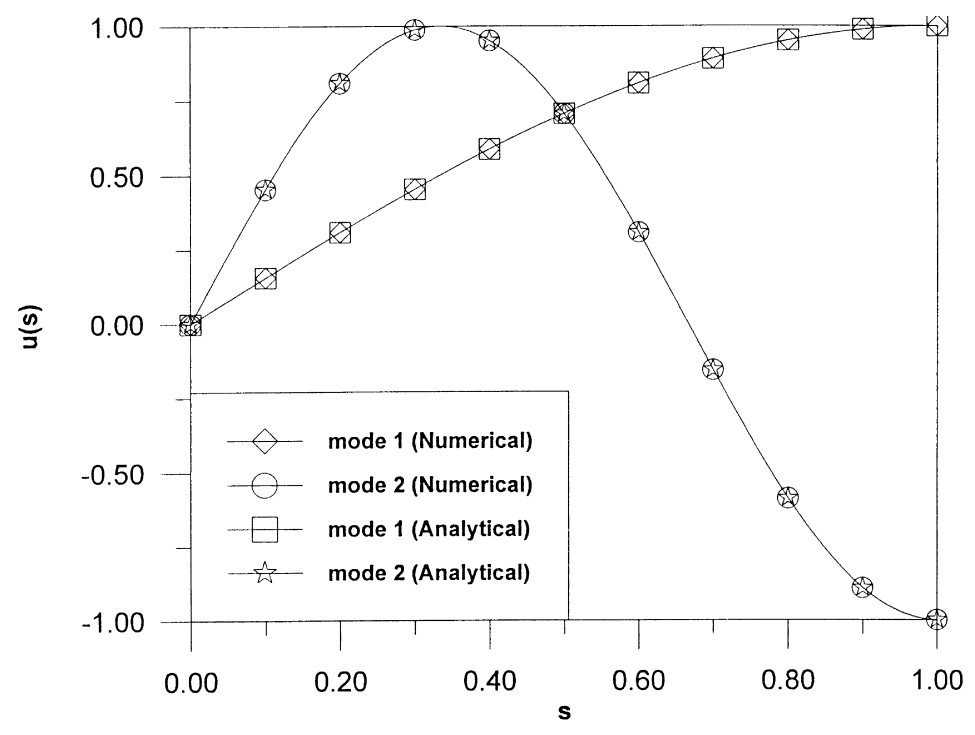

Fig. 6. The first two eigenmodes determined using the SVD method (Mixed B.C.: $u(0)=t(1)=0, n=20$ ).

\section{Conclusions}

In this article, we have used the dual equations for MRM to find the natural frequencies and modes of a rod. The SVD technique has been employed to distinguish whether the eigenvalue is true or not in the stage of eigenvalue searching. Also, the SVD method has been used to determine the true eigenvalues and the boundary eigenvectors at the same time, which can avoid calculating the spurious boundary eigenvector. Three examples with different boundary conditions have been used to show the validity of the present method.

\section{References}

[1] Niwa Y, Kobayashi S, Kitahara M. Determination of eigenvalue by boundary element methods Chap. 7. In: Banerjee PK, Shaw R, editors. Development in Boundary Element Methods, 2. New York: Appl. Sci. Pub, 1982.

[2] Beskos D, editor. Boundary Element Methods in Mechanics Amsterdam: North-Holland, 1987.

[3] Nowak AC, Neves AJ, editors. The Multiple Reciprocity Boundary Element Method Southampton: Comp.Mech. Publ, 1994.

[4] Nowak AJ, Brebbia CA. The multiple reciprocity method - a new approach for transforming BEM domain integrals to the boundary. Engineering Analysis with Boundary Elements 1989;6:164-167.

[5] Kamiya N, Andoh E. A note on multiple reciprocity integral formulation for Helmholtz equation. Communications in Numerical Methods in Engineering 1993;9:9-13.

[6] Chen JT, Wong FC. Analytical derivations for one-dimensional eigenproblems using dual BEM and MRM. Engineering Analysis with Boundary Elements 1997;20:25-33.

[7] Hong H-K, Chen JT. Derivation of integral equations in elasticity, J. Eng. Mech. Div. ASCE 1988;114:1028-1044.

[8] Chen JT, Hong H-K. Boundary element analysis and design in seepage flow problems with sheetpiles. Finite Elements in Analysis and Design 1994;17:1-20.

[9] Chen JT, Hong H-K. On the dual integral representation of boundary value problem in Laplace equation. Boundary Element Abstracts 1993;3:114-116.

[10] Kamiya N, Ando E, Nogae K. A new complex-valued formulation and eigenvalue analysis of the Helmholtz equation by boundary element method. Advances in Engineering Softwares 1996;26:219227.

[11] Yeih W, Chen JT, Chen KH, Wong FC. A. study on the multiple reciprocity method and complex-valued formulation for the Helmholtz equation. Advances in Engineering Softwares 1998;29:1-6.

[12] Press WH, Teukosky SA, Vetterling WT, Flannery BP. Numerical Recipes in FORTRAN. 2. New York: Cambridge University Press, 1992.

[13] Gloub GH, Van Loan CF. Matrix Computations. 2. Baltimore: The Johns Hopkins University Press, 1989. 